\title{
CONCERNING AUTOMORPHISMS OF NON-ASSOCIATIVE ALGEBRAS
}

\author{
R. D. SCHAFER
}

In their studies of non-associative algebras A. A. Albert and $\mathrm{N}$. Jacobson have made much use of the relationships which exist between an arbitrary non-associative algebra $\mathfrak{A}$ and its associative transformation algebra $T(\mathfrak{A})$. In this paper we are interested in the automorphism group $\mathbb{B}$ of $\mathfrak{A}$, and we sharpen the results of Jacobson $[3, \S 4]^{1}$ and Albert $[2, \S 9]$ in the sense that we prove $B$ isomorphic to a well-defined subgroup of the automorphism group of each of three associative algebras $(\$ \S 2,3)$.

Incidental to our proofs is the reconstruction (in the sense of equivalence) of an arbitrary non-associative algebra $\mathfrak{A}$ with unity element 1 from $T(\mathfrak{U})$ and from either of the enveloping algebras $E(R(\mathfrak{R}))$, $E(L(\mathfrak{A}))$ of respectively the right or left multiplications of $\mathfrak{A}$. This paper has been expanded in accordance with suggestions of the referee to include a more detailed study of the right ideals used in this reconstruction process $(\$ 5)$.

1. Preliminaries. Our notations are chiefly those of Albert as given in [1]. We regard a non-associative algebra $\mathfrak{A}$ of order $n$ over a field $\mathfrak{F}$ as consisting of a linear space $\mathfrak{R}$ of order $n$ over $\mathfrak{F}$, a linear space $R(\mathfrak{A})$ of linear transformations $R_{x}$ on $\mathfrak{R}$ of order $m \leqq n$ over $\mathfrak{F}$, and a linear mapping of $\mathbb{R}$ on $R(\mathfrak{A})$,

$$
x \rightarrow R_{x} .
$$

The elements $R_{x}$ of $R(\mathfrak{H})$ are called right multiplications, and $R(\mathfrak{H})$ the right multiplication space of $\mathfrak{A}$. Multiplication in $\mathfrak{A}$ is defined by

$$
a \cdot x=a R_{x} \text {. }
$$

The linearity of the right multiplications and of (1) insures distributivity in $\mathfrak{A}$ as well as the usual laws of scalar multiplication. We shall use the fact that, in case $\mathfrak{A}$ contains no absolute right divisor of zero (an element $x$ such that $a \cdot x=0$ for all $a$ in $\mathfrak{A}$ ), the mapping (1) is nonsingular and the order of $R(\mathfrak{T})$ over $\mathfrak{F}$ is $n$.

The linear transformations $L_{x}$ defined by

$$
a \rightarrow x \cdot a=a L_{x}
$$

Presented to the Society, August 23,1946; received by the editors August 2,1946, and, in revised form, November 13, 1946.

1 Numbers in brackets refer to the references cited at the end of the paper. 
are called left multiplications of $\mathfrak{A}$ and form the left multiplication space $L(\mathfrak{A})$ of $\mathfrak{A}$. The algebra $\mathfrak{A}$ may equally well be regarded as consisting of $\mathfrak{R}, L(\mathfrak{U})$, and the linear mapping

$$
x \rightarrow L_{x}
$$

of $\mathfrak{R}$ on $L(\mathfrak{A})$. Both $R(\mathfrak{A})$ and $L(\mathfrak{A})$ are linear subspaces of the total matric algebra $(\mathfrak{F})_{n}$ of all linear transformations on $\mathfrak{R}$.

If $\mathfrak{M}$ is a subset of $(\mathfrak{F})_{n}$, the algebra of all polynomials in the transformations in $\mathfrak{M}$ with coefficients in $\mathfrak{F}$ is called the enveloping algebra of $\mathfrak{M}$, and is denoted by $E(\mathfrak{M})$. We are particularly concerned with the enveloping algebras $E(R(\mathfrak{A}))$ and $E(L(\mathfrak{H}))$ of respectively the right and left multiplications of $\mathfrak{A}$, and with the transformation algebra $T(\mathfrak{A})=E(I, R(\mathfrak{A}), L(\mathfrak{H}))$ which is the algebra of all polynomials with coefficients in $\mathfrak{F}$ in the right and left multiplications of $\mathfrak{A}$ and the identity transformation $I$ in $(\mathfrak{F})_{n}$. We shall have occasion to write an arbitrary element $T$ of each of these algebras as follows:

$$
\begin{array}{lrl}
T=f\left(R_{x}, R_{y}, \cdots\right) & \text { for } T \text { in } E(R(\mathfrak{A})), \\
T=f\left(L_{x}, L_{y}, \cdots\right) & \text { for } T \text { in } E(L(\mathfrak{A})), \\
T=f\left(I, R_{x}, L_{x}, R_{y}, \cdots\right) & \text { for } T \text { in } T(\mathfrak{I}),
\end{array}
$$

where $x, y, \cdots$ are elements of $\mathfrak{A}$. In case $\mathfrak{A}$ contains a unity element 1 , then $R(\mathfrak{R})$ contains $I$, and we may write

$$
T=f\left(R_{x}, L_{x}, R_{y}, \cdots\right)
$$

for $T$ in $T(\mathfrak{R})$,

$x, y, \ldots$ in $\mathfrak{A}$.

If $\mathfrak{B}$ is a linear subspace of $\mathfrak{A}$, the set of all $R_{b}$ for $b$ in $\mathfrak{B}$ is a linear subspace $R(\mathfrak{B}, \mathfrak{X})$ of $R(\mathfrak{A})$, and the set of all $L_{b}$ is a linear subspace $L(\mathfrak{B}, \mathfrak{U})$ of $L(\mathfrak{R})$.

An automorphism $S$ of an algebra $\mathfrak{A}$ is a nonsingular linear transformation $x \rightarrow x S$ of $\mathfrak{A}$ on itself such that

$$
(a \cdot x) S=a S \cdot x S
$$

for all $a, x$ in $\mathfrak{A}$. In terms of right and left multiplications, (9) may be written equivalently as

$$
R_{x} S=S R_{x S}
$$

or

$$
L_{x} S=S L_{x S}
$$

for all $x$ in $\mathfrak{A}$. We shall use the facts that, if $S$ is an automorphism of $\mathfrak{A}$, then $S^{-1}$ is also, and if $\mathfrak{A}$ has a unity element 1 , then $1 S=1$. 
Inasmuch as the elements $T$ of subalgebras of $(\mathfrak{F})_{n}$ are themselves linear transformations, we shall denote linear transformations on subalgebras of $(\mathfrak{F})_{n}$ - such as $T(\mathfrak{Y}), E(R(\mathfrak{U})), E(L(\mathfrak{H}))$ - by Greek capitals, so that if $\Sigma$ is a linear transformation on $T(\mathfrak{A})$, say, we may write (without confusion) the image of $T$ under $\Sigma$ as $T \Sigma$.

An automorphism $S$ of $\mathfrak{A}$ determines an automorphism $\Sigma$ of $T(\mathfrak{A})$ as follows: let $T$ in $T(\mathfrak{A})$ be written in the form (7); then $\Sigma$ is the mapping

$$
T \rightarrow T \Sigma=f\left(I, R_{x S}, L_{x S}, R_{y S}, \cdots\right)=S^{-1} T S .
$$

For if $S$ is an automorphism of $\mathfrak{A}$, then $R_{x}=S R_{x S} S^{-1}, L_{x}=S L_{x S} S^{-1}$ by (10), (11), and $T=f\left(I, R_{x}, L_{x}, R_{y}, \cdots\right)=f\left(I, S R_{x S} S^{-1}, S L_{x S} S^{-1}\right.$, $\left.S R_{y S} S^{-1}, \cdots\right)=S f\left(I, R_{x S}, L_{x S}, R_{y S}, \cdots\right) S^{-1}=S(T \Sigma) S^{-1}$, or $T \Sigma$ $=S^{-1} T S$. The mapping (12) is obviously an automorphism of $T(\mathfrak{A})$. Moreover, $\Sigma$ induces automorphisms (which we do not distinguish notationally from $\Sigma$ ) on the subalgebras $E(R(\mathfrak{A})), E(L(\mathfrak{A}))$ of $T(\mathfrak{A})$ :

$$
\begin{array}{ll}
T \rightarrow T \Sigma=f\left(R_{x S}, R_{y S}, \cdots\right), & T \text { in } E(R(\mathfrak{U})) \text { as in (5), } \\
T \rightarrow T \Sigma=f\left(L_{x S}, L_{y S}, \cdots\right), & T \text { in } E(L(\mathfrak{U})) \text { as in (6). }
\end{array}
$$

If $S$ determines $\Sigma$ as in (12), then

$$
R(\mathfrak{U}) \Sigma=R(\mathfrak{I}), \quad L(\mathfrak{U}) \Sigma=L(\mathfrak{U}),
$$

since $R_{x} \Sigma=R_{x S}$ in $R(\mathfrak{A})$ while $L_{x} \Sigma=L_{x S}$ in $L(\mathfrak{U})$, and the nonsingularity of $\Sigma$ eliminates the possibility of proper inclusion.

2. Automorphisms of an algebra with unity element. Let $\mathfrak{A}$ be a non-associative algebra of order $n$ over $\mathfrak{F}$ with unity element 1 . We consider the elements of $\mathfrak{A}$ as comprising a linear space $\mathfrak{R}$ of order $n$ over $\mathfrak{F}$. Let $\mathfrak{B}$ be any (associative) algebra of linear transformations on $\mathfrak{R}$ which contains either $R(\mathfrak{A})$ or $L(\mathfrak{H})$. We intend to reconstruct $\mathfrak{A}$ (in the sense of equivalence) as an algebra of residue classes of $\mathfrak{B}$.

Denote by $\mathfrak{R}$ the set of all transformations $N$ in $\mathfrak{B}$ which annihilate 1 , that is, for which $1 N=0$. Then $\mathfrak{N}$ is a right ideal of $\mathfrak{B}$. For if $N, N_{1}$ are in $\mathfrak{R}$, then $1\left(\alpha N+\beta N_{1}\right)=\alpha 1 N+\beta 1 N_{1}=0$ for $\alpha, \beta$ in $\mathfrak{F}$, while $1 N T=0 T=0$ for any transformation $T$ in $\mathfrak{B}$. Denote by $\mathfrak{D}$ whichever set $R(\mathfrak{A})$ or $L(\mathfrak{U})$ is assumed to be contained in $\mathfrak{B}$, and by $D_{x}$ correspondingly the transformation $R_{x}$ or $L_{x}$. Then $\mathfrak{B}$ is the supplementary sum $\mathfrak{B}=\mathfrak{D}+\mathfrak{N}$. For $T$ in $\mathfrak{B}$ may be written uniquely in the form $T=D_{t}+N, 1 T=t, N$ in $\mathfrak{R}$.

Since $1 T N=t N$ which is not necessarily zero, $\mathfrak{N}$ is not in general a two-sided ideal of $\mathfrak{B}$ and we are not able to form the difference algebra $\mathfrak{B}-\mathfrak{N}$ when we take residue classes $[T]$ modulo $\mathfrak{N}$. Instead we form 
the difference group $\mathfrak{B}-\mathfrak{N}$ of residue classes $[T]$ modulo $\mathfrak{N}$ and have as usual a linear set over $\mathfrak{F}$ with respect to the operations $[T]+[U]$ $=[T+U], \lambda[T]=[\lambda T]$ of addition and scalar multiplication. Define multiplication in this linear set as follows:

$$
[T][U]=[X] \quad \text { where } 1 X=1 T \cdot 1 U,
$$

where the multiplication on the right is that in 2 . To see that for any $T, U$ in $\mathscr{B}$ such an $X$ exists, we need only to note that, if $X=D_{x}$ for $x=1 T \cdot 1 U$ in $\mathfrak{A}$, then $1 X=1 T \cdot 1 U$. This definition of multiplication is independent of the representatives $T, U$ since if $[T]=\left[T_{1}\right]$, $[U]=\left[U_{1}\right]$, then there exist $N, N_{1}$ in $\mathfrak{R}$ such that $T_{1}=T+N$, $U_{1}=U+N_{1}$, and $1 T_{1} \cdot 1 U_{1}=1(T+N) \cdot 1\left(U+N_{1}\right)=1 T \cdot 1 U$. With this multiplication the distributive laws hold in $\mathfrak{B}-\mathfrak{N}$. Hence $\mathfrak{B}-\mathfrak{N}$ is a non-associative algebra over $\mathfrak{F}$. Since there are no difference algebras used in this paper, there should be no confusion in the use of the notation $\mathfrak{B}-\mathfrak{R}$ for this algebra with multiplication defined by (16).

THEOREM 1. Let $\mathfrak{A}$ be a non-associative algebra over $\mathfrak{F}$ with unity element 1 , and $\mathfrak{B}$ be any (associative) algebra of linear transformations on $\mathfrak{A}$ containing either $R(\mathfrak{A})$ or $L(\mathfrak{H})$. If $\mathfrak{N}$ is the right ideal of transformations in $\mathfrak{B}$ annihilating 1 , then the non-associative algebra $\mathfrak{B}-\mathfrak{N}$ with multiplication defined by (16) is equivalent to $\mathfrak{A}$.

For in each residue class $[T]$ there is a unique transformation $D_{t}$ in $\mathfrak{D}(=R(\mathfrak{A})$ or $L(\mathfrak{H}))$ such that $1 T=1 D_{t}=t$. Then, since $\mathfrak{A}$ contains neither absolute right nor absolute left divisors of zero, the (obviously linear) mapping

$$
x \rightarrow D_{x} \rightarrow\left[D_{x}\right]
$$

is one-to-one on $\mathfrak{A}$ to $\mathfrak{B}-\mathfrak{N}$. But

$$
\left[D_{x}\right]\left[D_{y}\right]=\left[D_{x y}\right], \quad x, y \text { in } \mathfrak{A},
$$

since $x y=1 D_{x} \cdot 1 D_{y}=1 D_{x y}$. Then (17) is an equivalence of $\mathfrak{A}$ and $\mathfrak{B}-\mathfrak{N}$ since $x y \rightarrow D_{x y} \rightarrow\left[D_{x y}\right]=\left[D_{x}\right]\left[D_{y}\right]$ under (17).

Now $T(\mathfrak{A}), E(R(\mathfrak{A})), E(L(\mathfrak{A}))$ are among the algebras of linear transformations on the vector space $\mathbb{R}$ underlying $\mathfrak{A}$ which contain either $R(\mathfrak{A})$ or $L(\mathfrak{R})$-or both, as in the case of $T(\mathfrak{A})$ - and may be used as the algebra $\mathfrak{B}$ in Theorem 1 . We denote by $\mathfrak{N}_{T}$ the set of all $N$ in $T(\mathfrak{R})$ annihilating 1 and write $\mathfrak{N}_{R}=\mathfrak{N}_{T} \cap E(R(\mathfrak{R})), \mathfrak{R}_{L}=\mathfrak{N}_{T} \cap E(L(\mathfrak{R}))$. Then Theorem 1 implies that if multiplication in the respective algebras of residue classes is defined by (16) we have $\mathfrak{A} \cong T(\mathfrak{A})-\mathfrak{N}_{T}$ $\cong E(R(\mathfrak{U}))-\mathfrak{N}_{R} \cong E(L(\mathfrak{U}))-\mathfrak{N}_{L}$.

In the proof of the next theorem we must distinguish between the 
cases $\mathfrak{D}=R(\mathfrak{U})$ and $\mathfrak{D}=L(\mathfrak{U})$, and we use the following equations:

$$
\begin{array}{rlrl}
{\left[R_{x}\right]\left[R_{y}\right]} & =\left[R_{x y}\right]=\left[R_{x} R_{y}\right], & & x, y \text { in } \mathfrak{A}, \\
{\left[L_{x}\right]\left[L_{y}\right]} & =\left[L_{x y}\right]=\left[L_{y} L_{x}\right], & x, y \text { in } \mathfrak{A},
\end{array}
$$

verification of which is similar to that of (18).

ThEOREM 2. Let $\mathfrak{A}, \mathfrak{B}$, and $\mathfrak{N}$ be as in Theorem 1 , and $\mathfrak{D}$ be $R(\mathfrak{A})$ or $L(\mathfrak{A})$, whichever is assumed to be in $\mathfrak{B}$. If $\Sigma$ is an automorphism of $\mathfrak{B}$ such that $\mathfrak{N} \Sigma=\mathfrak{N}$ and $\mathfrak{D} \Sigma=\mathfrak{D}$, then $\Sigma$ determines an automorphism $S_{\Sigma}$ of $\mathfrak{A}$ as follows:

$$
S_{\Sigma}: x \rightarrow\left[D_{x}\right] \rightarrow\left[D_{x} \Sigma\right]=\left[D_{x^{\prime}}\right] \rightarrow x^{\prime}=x S_{\Sigma},
$$

for $x, x^{\prime}$ in $\mathfrak{A}$, where the $\left[D_{x}\right]$ are elements of $\mathfrak{B}-\mathfrak{N} \cong \mathfrak{A}$.

Note first that the mapping

$$
[T] \rightarrow[T \Sigma]
$$

of $\mathfrak{B}-\mathfrak{N}$ on itself is well-defined, since if $[T]=\left[T_{1}\right]$ then $T=T_{1}+N$

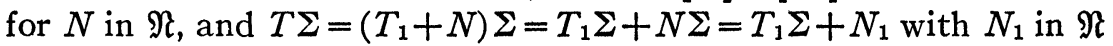
since $\mathfrak{R} \Sigma=\mathfrak{N}$. Hence $[T \Sigma]=\left[T_{1} \Sigma\right]$. Inasmuch as the correspondences $x \rightarrow\left[D_{x}\right]$ and $\left[D_{x^{\prime}}\right] \rightarrow x^{\prime}$ are equivalences between $\mathfrak{A}$ and $\mathfrak{B}-\mathfrak{N}$, we need only to show that (22) is an automorphism of $\mathfrak{B}-\mathfrak{N}$ in order to show that (21) is an automorphism of $\mathfrak{A}$. Now (22) is linear since $\alpha[T]+\beta[U]=[\alpha T+\beta U] \rightarrow[(\alpha T+\beta U) \Sigma]=[\alpha T \Sigma+\beta U \Sigma]=\alpha[T \Sigma]$ $+\beta[U \Sigma]$, and is nonsingular since $[T] \rightarrow[T \Sigma]=[0]$ implies $T \Sigma=N$ in $\mathfrak{N}, T=N \Sigma^{-1}=N_{1}$ in $\mathfrak{N},[T]=[0]$. Since $\mathfrak{D} \Sigma=\mathfrak{D}$, there exists an element $x_{1}$ of $\mathfrak{A}$ such that $D_{x} \Sigma=D_{x_{1}}$. But then $x_{1}=x^{\prime}$ since there is a unique transformation in $\mathfrak{D}$ in each residue class of $\mathfrak{B}$ modulo $\mathfrak{R}$. We may write $x^{\prime}=x S_{\Sigma}$ and

$$
D_{x} \Sigma=D_{x \mathcal{S}_{\Sigma}}
$$

We distinguish now between the cases $\mathfrak{D}=R(\mathfrak{A})$ and $\mathfrak{D}=L(\mathfrak{A})$. Let $\mathfrak{D}=R(\mathfrak{U})$ so that (19) holds. Then, since $\Sigma$ is an automorphism of $\mathfrak{B}$, we have $\left[R_{x}\right]\left[R_{y}\right]=\left[R_{x} R_{y}\right] \rightarrow\left[\left(R_{x} R_{y}\right) \Sigma\right]=\left[\left(R_{x} \Sigma\right)\left(R_{y} \Sigma\right)\right]=\left[R_{x S_{\Sigma}} R_{y S_{\Sigma}}\right]$ $=\left[R_{x S_{\Sigma}}\right]\left[R_{y S_{\Sigma}}\right]=\left[R_{x} \Sigma\right]\left[R_{y} \Sigma\right]$ under (22) which is an automorphism of $\mathfrak{B}-\mathfrak{N}$ as desired. In case $\mathfrak{D}=L(\mathfrak{A})$ it follows from (20) that $\left[L_{x}\right]\left[L_{y}\right]=\left[L_{y} L_{x}\right] \rightarrow\left[\left(L_{y} L_{x}\right) \Sigma\right]=\left[\left(L_{y} \Sigma\right)\left(L_{x} \Sigma\right)\right]=\left[L_{y S_{\Sigma}} L_{x S_{\Sigma}}\right]$ $=\left[L_{x S_{\Sigma}}\right]\left[L_{y S_{\Sigma}}\right]=\left[L_{x} \Sigma\right]\left[L_{y} \Sigma\right]$ under (22), completing the proof of the theorem.

We shall have occasion in the proof of the next theorem to use the fact that if $\mathfrak{B}$ contains both $R(\mathfrak{U})$ and $L(\mathfrak{A})$, and if both $R(\mathfrak{A})$ and $L(\mathfrak{A}) \longrightarrow$ as well of course as $\mathfrak{N}$-are their own images under an automorphism $\Sigma$ of $\mathfrak{B}$, then 


$$
R_{x} \Sigma=R_{x S_{\Sigma}}, \quad L_{x} \Sigma=L_{x S_{\Sigma}}
$$

for $S_{\Sigma}$ defined by (21).

ThEOREM 3. Let $\mathfrak{A}$ be a non-associative algebra with unity element 1 and automorphism group $(\$)$. Let $\mathfrak{S}_{T}$ be the group of automorphisms $\Sigma$ of $T(\mathfrak{H})$ such that $\mathfrak{N}_{T} \Sigma=\mathfrak{N}_{T}, R(\mathfrak{H}) \Sigma=R(\mathfrak{H}), L(\mathfrak{A}) \Sigma=L(\mathfrak{A})$. Then the correspondence $S \rightarrow \Sigma$ of (12) is an isomorphism of $\mathbb{S}$ onto $\mathfrak{W}_{T}$.

If $S$ is in $\$$ and $S \rightarrow \Sigma$ under (12), then $1(N \Sigma)=1 S^{-1} N S=1 N S=0 S$ $=0$ for $N$ in $\mathfrak{N}_{T}$. The nonsingularity of $\Sigma$ gives $\mathfrak{N}_{T} \Sigma=\mathfrak{N}_{T}$. By (15) we have $\Sigma$ in $\mathfrak{S}_{T}$. By Theorem 2 this $\Sigma$ determines an automorphism $S_{\Sigma}$ of $\mathfrak{A}$ :

$$
S_{\Sigma}: \quad x \rightarrow\left[R_{x}\right] \rightarrow\left[R_{x} \Sigma\right]=\left[R_{x S}\right] \rightarrow x S=x S_{\Sigma}
$$

for all $x$ in $\mathfrak{A}$, or $S=S_{\Sigma}$. Conversely, let $\Sigma$ be in $\mathfrak{S}_{T}$. Then $\Sigma$ determines an automorphism $S_{\Sigma}$ of $\mathfrak{A}$ which in turn determines an automorphism

$$
\Sigma_{*}: \quad T \rightarrow T \Sigma_{*}=S_{\Sigma}^{-1} T S_{\Sigma}, \quad T \text { in } T(\mathfrak{R}),
$$

of $T(\mathfrak{U})$ by (12). Write $T$ in the form (8). Then $T \Sigma_{*}=f\left(R_{x S_{\Sigma}}, L_{x S_{\Sigma}}\right.$, $\left.R_{y S_{\Sigma}}, \cdots\right)=f\left(R_{x} \Sigma, L_{x} \Sigma, R_{y} \Sigma, \cdots\right)=\left\{f\left(R_{x}, L_{x}, R_{y}, \cdots\right)\right\} \Sigma=T \Sigma$ by (12), (24), and the fact that $\Sigma$ is an automorphism of $T(\mathfrak{U})$. That is, $\Sigma_{*}=\Sigma$. It is clear then that $S \rightarrow \Sigma$ is a one-to-one mapping of (S) onto $\mathfrak{S}_{T}$. To see that $S \rightarrow \Sigma$ is an isomorphism we note only that if $S_{1}, S_{2}$ are in (S), $S_{1} \rightarrow \Sigma_{1}, S_{2} \rightarrow \Sigma_{2}$, then for $T$ in $T(\mathfrak{H})$ we have $T \Sigma_{1}=S_{1}^{-1} T S_{1}, T \Sigma_{1} \Sigma_{2}=S_{2}^{-1}\left(S_{1}^{-1} T S_{1}\right) S_{2}=\left(S_{1} S_{2}\right)^{-1} T\left(S_{1} S_{2}\right)$, or $S_{1} S_{2} \rightarrow \Sigma_{1} \Sigma_{2}$.

Variations in the proof of the following theorem from the proof above are trivial, consisting only of changes due to the fact that elements of $E(R(\mathfrak{A}))$ or $E(L(\mathfrak{A}))$ are generated by right or left multiplications alone.

THEOREM 4. The correspondences $S \rightarrow \Sigma$ of (13) and (14) are isomorphisms of $\mathbb{B}$ onto $\mathfrak{S}_{R}$ and $\mathfrak{S}_{L}$ respectively, where $\mathfrak{S}_{R}$ is the group of automorphisms $\Sigma$ of $E(R(\mathfrak{H}))$ such that $\mathfrak{N}_{R} \Sigma=\mathfrak{N}_{R}, R(\mathfrak{H}) \Sigma=R(\mathfrak{U})$, and $\mathfrak{S}_{L}$ is the group of automorphisms $\Sigma$ of $E\left(L(\mathfrak{R})\right.$ ) such that $\mathfrak{N}_{L} \Sigma=\mathfrak{N}_{L}$, $L(\mathfrak{R}) \Sigma=L(\mathfrak{R})$.

3. Automorphisms of an algebra without unity element. In case we are concerned with an algebra $\mathfrak{A}_{0}$ of order $(n-1)$ over $\mathfrak{F}$ without a unity element, we can easily modify the results of $\$ 2$ to include $\mathfrak{R}_{0}$. For we adjoin a unity element 1 to $\mathfrak{A}_{0}$ in the usual fashion to obtain an algebra $\mathfrak{A}$ of order $n$ over $\mathfrak{F}$ containing $\mathfrak{A}_{0}$ (in the sense of equivalence) as an ideal. Every element $x$ of $\mathfrak{A}$ may be written uniquely in the form 


$$
x=\xi 1+x_{0},
$$

$\xi$ in $\mathfrak{F}, x_{0}$ in $\mathfrak{A}_{0}$,

and if $y=\eta 1+y_{0}$, then $x+y=(\xi+\eta) 1+\left(x_{0}+y_{0}\right), \delta x=(\delta \xi) 1+\left(\delta x_{0}\right)$ for $\delta$ in $\mathfrak{F}, x y=(\xi \eta) 1+\left(\eta x_{0}+\xi y_{0}+x_{0} y_{0}\right)$. We shall write $\mathfrak{A}=1 \mathfrak{F}+\mathfrak{A}_{0}$ for the algebra so defined. Any automorphism $S_{0}$ of $\mathfrak{A}_{0}$ may be extended in a unique fashion to an automorphism $S$ of $\mathfrak{A}$ by defining

$$
S: \quad x \rightarrow x S=\xi 1+x_{0} S_{0},
$$

$x$ as in (26). Note that $S$ induces the automorphism $S_{0}$ within $\mathfrak{H}_{0}$.

It is apparent that an automorphism $S_{0}$ of $\mathfrak{A}_{0}$ determines a unique automorphism $\Sigma$ of $T(\mathfrak{U})$ as follows: $S_{0} \rightarrow S$ by (27), $S \rightarrow \Sigma$ by (12). Moreover, the linear subspaces $R\left(\mathfrak{A}_{0}, \mathfrak{U}\right)$ and $L\left(\mathfrak{H}_{0}, \mathfrak{H}\right)$ of $T(\mathfrak{H})$ are their own images under $\Sigma$. For if $x_{0}$ is in $\mathfrak{A}_{0}$, then $R_{x_{0}} \Sigma=R_{x_{0} S}=R_{x_{0} S_{0}}$ is in $R\left(\mathfrak{A}_{0}, \mathfrak{A}\right)$ and $L_{x_{0}} \Sigma=L_{x_{0} S}=L_{x_{0} S_{0}}$ is in $L\left(\mathfrak{A}_{0}, \mathfrak{A}\right)$.

If $\mathfrak{B}-\mathfrak{N}$ is the non-associative algebra equivalent to $\mathfrak{A}$ which was defined in $\$ 2$, then $\mathfrak{A}_{0}$ is equivalent to the ideal $\mathfrak{E}_{0}$ of $\mathfrak{B}-\mathfrak{N}$ consisting of residue classes $\left[D_{x_{0}}\right]$ for $x_{0}$ in $\mathfrak{A}_{0}$, that is, for $D_{x_{0}}$ in $\mathfrak{D}_{0}=R\left(\mathfrak{H}_{0}, \mathfrak{A}\right)$ or $L\left(\mathfrak{A}_{0}, \mathfrak{A}\right)$ according as $\mathfrak{D}=R(\mathfrak{A})$ or $L(\mathfrak{A})$. For, by Theorem $1, \mathfrak{A}$ is isomorphic to $\mathfrak{B}-\mathfrak{N}$ under the mapping (17). Since $\mathfrak{A}_{0}$ is an ideal of $\mathfrak{A}$, the mapping

$$
x_{0} \rightarrow\left[D_{x_{0}}\right],
$$

$x_{0}$ in $\mathfrak{A}_{0}$,

determines an ideal $\mathfrak{S}_{0}$ of $\mathfrak{B}-\mathfrak{R}$, and $\mathfrak{\mho}_{0} \cong \mathfrak{A}_{0}$.

Let $\Sigma$ be an automorphism of $\mathfrak{B}$ such that $\mathfrak{R} \Sigma=\mathfrak{N}$ and $\mathfrak{D}_{0} \Sigma=\mathfrak{D}_{0}$. Then $\Sigma$ determines an automorphism $S_{0 \Sigma}$ of $\mathfrak{R}_{0}$ as follows:

$$
S_{0 \Sigma}: \quad x_{0} \rightarrow\left[D_{x_{0}}\right] \rightarrow\left[D_{x_{0}} \Sigma\right]=\left[D_{x_{0}^{\prime}}\right] \rightarrow x_{0}^{\prime}=x_{0} S_{0 \Sigma},
$$

for $x_{0}, x_{0}^{\prime}$ in $\mathfrak{A}_{0}$. For $\mathfrak{D}=I \mathfrak{F}+\mathfrak{D}_{0}$, and any automorphism of $\mathfrak{B}$ leaves invariant the subspace $I \mathscr{F}$ of order 1 , so that $\mathfrak{D} \Sigma=\mathfrak{D}$. Then by Theorem $2, \Sigma$ determines an automorphism $S_{\Sigma}$ of $\mathfrak{A}$. But $S_{\Sigma}$ induces on $\mathfrak{A}_{0}$ the automorphism (29) since $D_{x_{0}} \Sigma=D_{x_{0}^{\prime}}$ in $\mathfrak{D}_{0}$ implies $x_{0}^{\prime}$ is in $\mathfrak{A}_{0}$. Thus $x_{0} \rightarrow\left[D_{x_{0}}\right] \rightarrow\left[D_{x_{0}} \Sigma\right]=\left[D_{x_{0}^{\prime}}\right] \rightarrow x_{0}^{\prime}=x_{0} S_{\Sigma}$ is in $\mathfrak{A}_{0}$, or $S_{\Sigma}$ induces $S_{0 \Sigma}$ on $\mathfrak{A}_{0}$.

THEOREM 5. Let $\mathfrak{A}_{0}$ be a non-associative algebra without unity element, and let $\mathfrak{A}=1 \mathfrak{F}+\mathfrak{H}_{0}$. Let $\mathfrak{S}_{\mathrm{T}}^{0}$ be the group of automorphisms $\Sigma$ of $T(\mathfrak{H})$ such that $R\left(\mathfrak{A}_{0}, \mathfrak{A}\right) \Sigma=R\left(\mathfrak{A}_{0}, \mathfrak{A}\right), L\left(\mathfrak{H}_{0}, \mathfrak{A}\right) \Sigma=L\left(\mathfrak{H}_{0}, \mathfrak{N}\right), \mathfrak{N}_{T} \Sigma=\mathfrak{N}_{T}$. Then the correspondence $S_{0} \rightarrow S \rightarrow \Sigma$ of (27) and (12) is an isomorphism of the automorphism group $\mathfrak{S H}_{0}$ of $\mathfrak{A}_{0}$ onto $\mathfrak{S}_{T}^{0}$.

For if $S_{0}$ is in $\mathfrak{S}_{0}$, then $S_{0} \rightarrow S \rightarrow \Sigma$ in $\mathfrak{S}_{T}^{0}$ and $\Sigma \rightarrow S_{\Sigma}=S$ by Theorem 3. But then $S$ induces the automorphism $S_{0 \Sigma}$ within $\mathfrak{A}_{0}$. That is, $S_{0 \Sigma}=S_{0}$. Conversely, if $\Sigma$ is in $\mathfrak{S}_{T}^{0}$, then $\Sigma \rightarrow S_{0 \Sigma}$ in $\oiint_{0}$ by (29). But $S_{0 \Sigma} \rightarrow S_{\Sigma} \rightarrow \Sigma_{*}$ by (27) and (12) and $\Sigma_{*}=\Sigma$ by Theorem 3 . Hence the 
mapping $S_{0} \rightarrow S \rightarrow \Sigma$ of $\oiint_{0}$ on $\mathfrak{\Phi}_{T}^{0}$ is one-to-one, and is by Theorem 3 an isomorphism.

The results analogous to Theorem 4 for algebras $\mathfrak{A}_{0}$ without unity quantity may be stated as follows: let $\mathfrak{S}_{R}^{0}$ be the group of automorphisms $\Sigma$ of $E(R(\mathfrak{A}))$ such that $R\left(\mathfrak{A}_{0}, \mathfrak{A}\right) \Sigma=R\left(\mathfrak{A}_{0}, \mathfrak{A}\right), \mathfrak{N}_{R} \Sigma=\mathfrak{N}_{R}$. Then the correspondence $S_{0} \rightarrow S \rightarrow \Sigma$ of (27) and (13) is an isomorphism of the automorphism group $\mathfrak{S}_{0}$ of $\mathfrak{U}_{0}$ onto $\mathfrak{S}_{R}^{0}$. Let $\mathfrak{W}_{L}^{0}$ be the group of automorphisms $\Sigma$ of $E(L(\mathfrak{U}))$ such that $L\left(\mathfrak{A}_{0}, \mathfrak{U}\right) \Sigma=L\left(\mathfrak{A}_{0}, \mathfrak{U}\right)$, $\mathfrak{N}_{L} \Sigma=\mathfrak{N}_{L}$. Then the correspondence $S_{0} \rightarrow S \rightarrow \Sigma$ of (27) and (14) is an isomorphism of $\mathfrak{S}_{0}$ onto $\mathfrak{S}_{L}^{0}$.

4. Inner automorphisms $\Sigma$ of $T(\mathfrak{A})$. An automorphism $\Sigma$ of the associative algebra $T(\mathfrak{A})$ is called inner in case $T \rightarrow T \Sigma=K^{-1} T K$ for some nonsingular element $K$ of $T(\mathfrak{U})$. We are concerned in this section with automorphisms $S$ of $\mathfrak{A}$ which determine inner automorphisms $\Sigma$ of $T(\mathfrak{U})$ under (12).

The group $\Re$ of all inner automorphisms of $T(\mathfrak{A})$ is an invariant subgroup of the automorphism group of $T(\mathfrak{A})$. If $\mathfrak{S}_{T}$ is the group of automorphisms of $T(\mathfrak{U})$ described in Theorem 3 , then the intersection $\mathfrak{S}_{T} \cap \mathfrak{\Omega}$ is an invariant subgroup of $\mathfrak{E}_{\boldsymbol{T}}$. But then there is an invariant subgroup $\Im$ of the automorphism group $\mathbb{H}$ of $\mathfrak{A}$ such that $\mathfrak{\Im} \cong \mathfrak{W}_{T} \cap \Re$ under the correspondence $S \rightarrow \Sigma$ of (12). The elements of $\Im$ are characterized as those automorphisms of $\mathfrak{A}$ which are themselves elements of $T(\mathfrak{U})$ by

THEOREM 6. Let $\mathfrak{A}$ be a non-associative algebra over $\mathfrak{F}$ with unity element 1 and automorphism $S$ determining an automorphism $\Sigma$ of $T(\mathfrak{A})$ by (12). Then $\Sigma$ is inner if and only if $S$ is in $T(\mathfrak{A})$.

If $S$ is in $T(\mathfrak{A})$, then $T \rightarrow T \Sigma=S^{-1} T S$ is an inner automorphism of $T(\mathfrak{U})$. Conversely, if $\Sigma$ is inner, there exists a nonsingular element $K$ of $T(\mathfrak{H})$ such that $T \Sigma=K^{-1} T K$ for all $T$ in $T(\mathfrak{U})$. In particular, $R_{x S}=R_{x} \Sigma=K^{-1} R_{x} K$. Let $1 K=k$ so that $x S L_{k}=k \cdot x S=k R_{x \rightarrow S}$ $=1 K K^{-1} R_{x} K=x K$ for all $x$ in $\mathfrak{A}$, or $S L_{k}=K$. Since $S$ and $K$ are nonsingular, $L_{k}^{-1}$ exists. Moreover, $L_{k}^{-1}$ is in $T(\mathfrak{U})$, and $S=K L_{k}^{-1}$ is in $T(\mathfrak{I})$.

Perhaps it should be pointed out that Theorem 6 yields nothing in the case of central simple algebras (that is, algebras which are simple for all scalar extensions). For although it is true that, if $\mathfrak{A}$ is central simple, then $T(\mathfrak{A})$ is also and-by a well known theorem concerning associative algebras-every automorphism $\Sigma$ of $T(\mathfrak{H})$ is inner, so that Theorem 6 implies that every automorphism $S$ of $\mathfrak{A}$ is in $T(\mathfrak{A})$, it is also true $[1, \mathfrak{8} 8]$ that in this case $T(\mathfrak{A})=(\mathfrak{F})_{n}$, the algebra 
of all linear transformations on $\mathfrak{A}$. Of course it is vacuous then to say that $S$ is in $T(\mathfrak{U})$.

5. The right ideals $\mathfrak{N}_{T}, \mathfrak{N}_{R}, \mathfrak{N}_{L}$. We now make a more thorough analysis of the right ideals $\mathfrak{N}_{T}, \mathfrak{N}_{R}, \mathfrak{N}_{L}$ of $T(\mathfrak{U}), E(R(\mathfrak{A})), E(L(\mathfrak{U}))$, respectively, and arrive in particular at criteria for the (right, left) simplicity of an algebra $\mathfrak{A}$ with unity quantity.

THEOREM 7. An algebra $\mathfrak{A}$ with unity quantity is both commutative and associative if and only if $\mathfrak{N}_{T}=0$.

For $\mathfrak{N}_{T}=0$ implies that $L_{x}-R_{x}=R_{x} R_{y}-R_{x y}=0$ for all $x, y$ in $\mathfrak{A}$. That is,

$$
R_{x}=L_{x}, \quad R_{x} R_{y}=R_{x y},
$$

$\mathfrak{A}$ is both commutative and associative. Conversely, if (30) holds for all $x, y$ in $\mathfrak{A}$, then $T$ in $T(\mathfrak{U})$ has the form $T=f\left(R_{x}, L_{x}, R_{y}, \cdots\right)$ $\left.=g\left(R_{x}, R_{y}, \cdots\right)=R_{g(x, y}, \cdots\right)$. Then $1 T=0$ implies $g(x, y, \cdots)=0$ or $T=0$. Hence $\mathfrak{N}_{T}=0$.

The center $\mathbb{Z}$ of $\mathfrak{A}$ consists of all elements $c$ in $\mathfrak{A}$ such that

$$
x c=c x, \quad c(x y)=(c x) y=x(c y),
$$

or equivalently

$$
c L_{x}=c R_{x}, \quad c R_{x y}=c R_{x} R_{y}=c R_{y} L_{x}
$$

for all $x, y$ in $\mathfrak{A}$.

THEOREM 8. An element $c$ is in the center 3 of an algebra $\mathfrak{A}$ with unity quantity if and only if $c \mathfrak{N}_{T}=0$.

Certainly $L_{x}-R_{x}, R_{x y}-R_{x} R_{y}, R_{x y}-R_{y} L_{x}$ are in $\mathfrak{N}_{T}$ for all $x, y$ in $\mathfrak{A}$. Hence if $c \mathfrak{N}_{T}=0$, it follows that $c\left(L_{x}-R_{x}\right)=c\left(R_{x y}-R_{x} R_{y}\right)$ $=c\left(R_{x y}-R_{y} L_{x}\right)=0$ or (32) holds, $c$ is in the center of $\mathfrak{A}$. Conversely, if $c$ is in the center of $\mathfrak{A}$, and if we write $T$ in $T(\mathfrak{U})$ as in (8), it is seen by repeated application of (32) that $c T=c f\left(R_{x}, L_{x}, R_{y}, \cdots\right)$ $=c R_{g(x, y, \cdots)}$ where the non-associative polynomial $g(x, y, \cdots)$ $=1 f\left(R_{x}, L_{x}, R_{y}, \cdots\right)=1 T$. But if $T$ is in $\mathfrak{N}_{T}$, then $1 T=0$ so that $g(x, y, \cdots)=0$ and $c T=0, c \mathfrak{N}_{T}=0$.

An algebra $\mathfrak{A}$, which is not the zero algebra of order 1 , is called simple (right simple, left simple) in case the only ideals (right ideals, left ideals) of $\mathfrak{A}$ are 0 and $\mathfrak{A}$.

THEOREM 9. A non-associative algebra $\mathfrak{A}$ with unity quantity is right simple if and only if $\mathfrak{N}_{R}$ is a maximal proper right ideal of $E(R(\mathfrak{A})$ ).

If $\mathfrak{N}_{R}$ is a maximal proper right ideal of $E(R(\mathfrak{U}))$, then the only 
right ideal of $E(R(\mathfrak{A}))$ containing $\mathfrak{N}_{R}$ properly is $E(R(\mathfrak{U}))$ itself. We assume that $\mathfrak{A}$ is not right simple, so that $\mathfrak{A}$ has a right ideal $\mathfrak{Q} \neq 0$, $\mathfrak{A}$. Let $\mathfrak{P}$ be the linear set $\mathfrak{P}=R(\mathfrak{Q}, \mathfrak{A})+\mathfrak{N}_{R}$. Then $P$ in $\mathfrak{P}$ has the form $P=R_{q}+N, q$ in $\mathfrak{Q}, N$ in $\mathfrak{N}_{R}$, and any element $T$ of $E(R(\mathfrak{U}))$ may be written as $T=R_{t}+N_{1}, t$ in $\mathfrak{A}, N_{1}$ in $\mathfrak{N}_{R}$, so that $P T$ $=\left(R_{q}+N\right)\left(R_{t}+N_{1}\right)=R_{q} R_{t}+R_{q} N_{1}+N T=R_{q t}+\left(R_{q} R_{t}-R_{q t}\right)+R_{q} N_{1}$ $+N T$. Now $R_{q} N_{1}=R_{a}+N_{2}$ for $a$ in $\mathfrak{A}, N_{2}$ in $\mathfrak{N}_{R}$, and $1 R_{q} N_{1}=1 R_{a}$ $+1 N_{2}$ or $a=q N_{1}$. Since $N_{1}=f\left(R_{x}, R_{y}, \cdots\right)$ while $\mathfrak{Q}$ is a right ideal of $\mathfrak{A}$, it follows that $a=q N_{1}=q f\left(R_{x}, R_{y}, \cdots\right)$ is in $\mathfrak{Q}$. Hence $P T=R_{q t+q N_{1}}+\left(R_{q} R_{t}-R_{q t}+N_{2}+N T\right)$ is in $\mathfrak{B}$ since $q t+q N_{1}$ is in $\mathfrak{Q}$ while $R_{q} R_{t}-R_{q t}+N_{2}+N T$ is in $\mathfrak{R}_{R}$. Hence $\mathfrak{P}$ is a right ideal of $E(R(\mathfrak{A}))$ containing $\mathfrak{N}_{R}$. Since $\mathfrak{Q} \neq 0, \mathfrak{A}$, it follows that $R(\mathfrak{Q}, \mathfrak{A})$, being of the same dimension over $\mathfrak{F}$ as $\mathfrak{Q}$, is neither 0 nor $R(\mathfrak{U})$, and then $\mathfrak{P} \neq \mathfrak{N}_{R}, E(R(\mathfrak{U}))$, a contradiction. Hence $\mathfrak{A}$ is right simple.

Conversely, let $\mathfrak{P}$ be any proper right ideal of $E(R(\mathfrak{A}))$ which contains $\mathfrak{N}_{R}$. Consider the set $\mathfrak{Q}$ of residue classes $[P]$ modulo $\mathfrak{N}_{R}$ for $P$ in $\mathfrak{B}$. Then $\mathfrak{Q}$ is a linear subset of $E(R(\mathfrak{U}))-\mathfrak{N}_{R} \cong \mathfrak{A}$. Moreover, if $[P]$ is any element of $\mathfrak{Q}$, we write $P=R_{p}+N$ for $p$ in $\mathfrak{A}, N$ in $\mathfrak{N}_{R}$. Let $\left[R_{t}\right]$ be any element of $E(R(\mathfrak{U}))-\mathfrak{N}_{R}$. Then $P R_{t}=R_{p} R_{t}+N R_{t}$ $=P_{1}$ in $\mathfrak{P}$ since $\mathfrak{P}$ is a right ideal of $E(R(\mathfrak{U}))$. Then

$$
[P]\left[R_{t}\right]=\left[R_{p}\right]\left[R_{t}\right]=\left[R_{p} R_{t}\right]=\left[P_{1}\right]
$$

in $\mathfrak{O}$ by (19), and $\mathfrak{O}$ is a right ideal of $E(R(\mathfrak{U}))-\mathfrak{N}_{R} \cong \mathfrak{A}$. If $\mathfrak{A}$ is right simple, then either $\mathfrak{Q}=[0]$ or $\mathfrak{Q}=E(R(\mathfrak{U}))-\mathfrak{N}_{R}$. In the latter case, $\mathfrak{Q}$ contains $[I], \mathfrak{B}$ contains $I+N_{1}$ for some $N_{1}$ in $\mathfrak{N}_{R}$. Since $\mathfrak{P}$ also contains $N_{1}$, it follows that $I$ is in $\mathfrak{B}$, whence $\mathfrak{P}=E(R(\mathfrak{A}))$, a contradiction. Hence $\mathfrak{Q}=[0], \mathfrak{P}=\mathfrak{N}_{R}$, and $\mathfrak{N}_{R}$ is a maximal proper right ideal of $E(R(\mathfrak{U}))$.

An exactly symmetrical argument, involving left multiplications instead of right multiplications, suffices to prove

THEOREM 10. A non-associative algebra $\mathfrak{A}$ with unity quantity is left simple if and only if $\mathfrak{N}_{L}$ is a maximal proper right ideal of $E(L(\mathfrak{N})$ ).

Only obvious variations on the proof above are required in the proof of

THEOREM 11. A non-associative algebra $\mathfrak{O}$ with unity quantity is simple if and only if $\mathfrak{N}_{T}$ is a maximal proper right ideal of $T(\mathfrak{U})$.

For example, to prove the converse part of the theorem, we let $\mathfrak{P}$ be any proper right ideal of $T(\mathfrak{A})$ which contains $\mathfrak{N}_{T}$, and let $\mathfrak{Q}$ be the linear space of residue classes $[P]$ modulo $\mathfrak{N}_{T}$ for $P$ in $\mathfrak{P}$. We may write $P=R_{p}+N=L_{p}+N_{0}$ for $N, N_{0}$ in $\mathfrak{N}_{T}$, and let $\left[R_{t}\right]=\left[L_{t}\right]$ be 
any element of $T(\mathfrak{U})-\mathfrak{N}_{T}$. Then we have (33) as before, where now the quantities involved are residue classes of $T(\mathfrak{A})$ modulo $\mathfrak{N}_{T}$, but also we have $P L_{t}=L_{p} L_{t}+N_{0} L_{t}=P_{2}$ in $\mathfrak{P}$ so that $\left[L_{t}\right][P]=\left[L_{t}\right]\left[L_{p}\right]$ $=\left[L_{p} L_{t}\right]=\left[P_{2}\right]$ in $\mathfrak{Q}$ by $(20)$, and $\mathfrak{Q}$ is an ideal of $T(\mathfrak{A})-\mathfrak{N}_{T} \cong \mathfrak{A}$. The remainder of the proof is as before.

We conclude with an analysis of the structure of the right ideal $\mathfrak{N}_{T}$ of $T(\mathfrak{U})$ in case $\mathfrak{A}$ of order $n$ over $\mathfrak{F}$ (with unity quantity) is simple. In this case $T(\mathfrak{A})=(\mathfrak{B})_{8}$ where the center $\mathbb{Z}$ of $\mathfrak{A}$ is a field of degree $t$ over $\mathfrak{F}$, and $n=s t$ (see $[1, \S \S 8,19])$.

THEOREM 12. Let $\mathfrak{A}$ be a simple non-associative algebra of order $n=s t$ over $\mathfrak{F}$ with unity quantity and with center $\mathbb{B}$ of degree $t$ over $\mathfrak{F}$. Then $\mathfrak{N}_{T}=\Re+(\mathfrak{3})_{s-1}$, where the radical $\mathfrak{R}$ of $\mathfrak{N}_{T}$ has order $(s-1)$ over $\mathbb{B}$ and the semi-simple component of $\mathfrak{N}_{T}$ is the total matric algebra $(\mathfrak{B})_{8-1}$ of degree $(s-1)$ over 3 .

For $\mathfrak{A}$ is central simple over $\mathfrak{Z}$. Let $\left(1, u_{2}, \cdots, u_{s}\right)$ be a fixed basis of $\mathfrak{A}$ over $\mathfrak{B}$. Then, since $T(\mathfrak{A})=(\mathfrak{B})_{8}$, it follows from Theorem 8 that $\mathfrak{N}_{T}$ (over $\mathbb{B}$ ) consists of all $s$-by-s matrices with first row zero. But the structure of this algebra of matrices, with principal idempotent

$$
E=\left(\begin{array}{ll}
0 & 0 \\
0 & I_{s-1}
\end{array}\right),
$$

is easily determined. Its radical $\Re$ consists of all matrices (with elements in 3) of the form

$$
\left(\begin{array}{ll}
0 & 0 \\
U & 0
\end{array}\right)
$$

where $U$ is any $(s-1)$-by-1 matrix. Its semi-simple component consists of all matrices (with elements in 3) of the form

$$
\left(\begin{array}{ll}
0 & 0 \\
0 & V
\end{array}\right)
$$

where $V$ is any $(s-1)$-rowed square matrix. This is a total matric algebra $(\boldsymbol{B})_{s-1}$.

\section{REFERENCES}

1. A. A. Albert, Non-associative algebras. I. Fundamental concepts and isotopy, Ann. of Math. (2) vol. 43 (1942) pp. 685-708.

2. - Quasigroups. II. Trans. Amer. Math. Soc. vol. 55 (1944) pp. 401-419.

3. N. Jacobson, $A$ note on non-associative algebras, Duke Math. J. vol. 3 (1937) pp. 544-548.

The Institute for Advanced Study 